\title{
Construcción de la flexibilidad en una universidad colombiana pública a distancia con modalidad virtual
}

\section{The Construction of Flexibility in a Virtual Program at a Public Colombian Distance Education University}

\author{
René Montero Vargas \\ Centro de Investigaciones y Estudios Avanzados de Instituto Politécnico Nacional de México. Ciudad de México. \\ México. \\ monterorene@gmail.com \\ Judith Kalman \\ Centro de Investigaciones y Estudios Avanzados de Instituto Politécnico Nacional de México. Ciudad de México. \\ México. \\ jkalman@ cinvestav.mx
}

\begin{abstract}
Resumen
La flexibilidad en un programa en línea de educación superior se refiere a un concepto formulado por organismos internacionales y reproducido por diversas instituciones. Esta noción propone que los estudiantes tienen múltiples opciones de tiempo, espacio y metodologías para realizar las actividades del curso. Sin embargo, hay pocos estudios que se centran en las formas o maneras en que los estudiantes interpretan este concepto en los programas en línea. Este estudio cualitativo describe algunas de estas formas y analiza las participaciones de los estudiantes en un foro en línea utilizado para desarrollar actividades de aprendizaje en el contexto de un curso que se realiza en línea en una plataforma Moodle. Nuestros resultados muestran algunas de las formas en que la flexibilidad se construye en la práctica en dos grupos de estudiantes mientras interactúan en un aula digital.
\end{abstract}

\section{Palabras clave.}

Educación a distancia, educación superior, flexibilidad de la educación, análisis del discurso.

\begin{abstract}
Flexibility in an online program of higher education, refers a concept formulated by international organisms and reproduced by diverse institutions. This notion proposes that students have multiple options in time, space, and methodologies to realize course activities. However, there are few studies that focus on the ways or forms in which this concept is construed by students in online programs. This qualitative study describes some of these ways and analyzes students' participations in an online forum used to develop learning activities within the context of a course being taken online on a Moodle platform. Our results show some of the ways that flexibility plays out in practice in two groups of students as they interact in online classrooms practices.
\end{abstract}

Keywords

Distance education, higher education, educative flexibility, discourse analysis. 


\section{Introducción}

Este artículo ofrece un acercamiento a los modos en que un grupo de estudiantes y su maestra construyen el significado de la flexibilidad del tiempo, en los procesos de formación en un curso de educación a distancia. Para ello, se reconoce que los organismos oficiales, nacionales e internacionales, promueven los procesos de formación en esta modalidad educativa argumentando que la educación a distancia permite superar las dificultades de tiempo y espacio que impone la educación presencial para quienes deben llevar modos de vida donde coexisten las actividades laborales y académicas (García, 2009).

Por citar un ejemplo, la Organización para la Cooperación y el Desarrollo EconómicoOCDE (2015), afirma que la flexibilidad de tiempo hace que la educación a distancia resulte una opción apropiada para adultos con compromisos laborales y personales, lo cual dificulta contar con tiempo para desarrollar programas educativos presenciales.

La UNESCO, por su parte, también se ha ocupado de promover la educación a distancia empleando la flexibilidad como argumento. Al respecto, señala que esta modalidad educativa "permite un manejo flexible del tiempo de aprendizaje y duración, ritmo e intensidad de estudio, de acuerdo con las características de los usuarios y de los contenidos" (Maya, 1993; 27).

En su referencia, al igual que la OCDE, UNESCO señala que "para el estudiante, el aprendizaje abierto y a distancia constituye un aumento de la flexibilidad en el acceso a la educación, y permite combinar educación y trabajo" (2002;12). De esta forma, la UNESCO ratifica que estudiar a distancia ofrece la posibilidad de combinar los tiempos para desarrollar actividades laborales y educativas, pues enfatiza específicamente que las ofertas de formación académica que se desarrollan bajo la modalidad a distancia ofrecen "flexibilidad en tiempo, lugar, ritmo y métodos de estudio de los usuarios" (Concha, 2014; 6).

Estas aproximaciones revelan la coincidencia entre las ideas de estas instituciones sobre las bondades de la educación a distancia, y dejan ver sobre todo una mirada de la flexibilidad enfocada en el manejo del tiempo del estudiante. No obstante, en ninguna de las formulaciones presentadas se encuentra una definición concreta de lo que significa la flexibilidad, más allá de realizar las actividades a conveniencia del tiempo de los educandos. Esta situación deja abierta la posibilidad de indagar y documentar las formas en que los participantes interactúan y se organizan para realizar las actividades que les asignan los maestros en sus programas académicos.

Nos referimos específicamente a que esta idea de que el estudiante tiene la posibilidad de decidir sobre el uso del tiempo para el desarrollo de las tareas, fundamentada en "el principio de flexibilidad, en función de cuándo y cómo el alumno estudia" (UNESCO, 2017), contrasta con situaciones como las que vamos a analizar. En nuestros datos, un estudiante de un grupo se presenta con sus compañeros faltando dos días para entregar un trabajo, comparte su tarea terminada completamente y, al margen de haber cumplido con lo solicitado por la maestra y haber intervenido en el foro dentro del tiempo habilitado para construir la tarea, se encuentra con que los demás integrantes del equipo desconocen su trabajo en el documento final enviado a la profesora. Esta situación se pudo haber debido a que el estudiante ingresó su tarea cuando los demás ya habían

Construcción de la flexibilidad en una universidad colombiana pública a distancia con modalidad virtual. René Montero y Judith Kalman. 
elaborado el producto final o a que el estudiante no participó en el foro, ni entregó avances con la misma frecuencia que los demás.

Desde esta perspectiva, la idea de una educación para todos promovida por la UNESCO (2002), donde las personas cuenten con acceso a programas de formación aun cuando deban compartir espacios laborales y formativos, permite investigar las formas en que se construye el carácter flexible de la educación a distancia en la práctica, es decir, directamente desde las interacciones entre los estudiantes y sus profesores.

En este sentido, proponemos aportar al conocimiento sobre las posibilidades y limitaciones que puede ofrecer lo flexible de esta modalidad educativa, toda vez que se constituye en una de las consignas más fuertes que usan los organismos internacionales y las instituciones para promover esta modalidad de formación.

\section{Aproximaciones teóricas}

Para desarrollar el análisis exploramos las formas en que se vive la flexibilidad en términos del uso del tiempo, desde las interacciones entre los participantes del aula en línea. Analizamos los turnos conversacionales en un foro en línea de la plataforma Moodle, de un grupo de cinco estudiantes que desarrollan las tareas de una de las unidades temáticas de un curso de Biología Ambiental, en un programa de formación a distancia en modalidad virtual en una universidad pública colombiana.

El abordaje teórico que empleamos retoma de la teoría sociocultural el carácter situado de los eventos (Lave \& Wenger, 1991; Wertsh, 1993), por cuanto suceden en contextos y situaciones particulares que se construyen a través de interacciones entre los participantes, en este caso los estudiantes, la maestra del curso y la institución. Así mismo, partimos de que estas interacciones están mediadas, cuando menos, por los diseños curriculares de los programas, las consignas de las actividades que proponen los maestros para cada unidad temática de los cursos y las formas en que se evalúan los procesos de aprendizaje.

Para nosotros resultó necesario hacer un acercamiento directamente a los registros en el foro en línea de la plataforma de la Universidad, es decir partir de la evidencia empírica, para elaborar las descripciones (Blommaert \& Dong, 2010), que nos permiten reconstruir los significados que construyeron los participantes (Heath, 1982), en este caso sobre la flexibilidad en términos de uso del tiempo para estudiar, y así aportar elementos a las discusiones sobre esta modalidad educativa que se define como participativa, inclusiva, motivadora colaborativa y, por supuesto flexible (Cabero, 2016).

Dado que los datos que se recogieron de los foros de trabajo son mensajes que escribieron los participantes, fue necesario adentrarnos en el análisis del discurso escrito (Blommaert, 2005; Hernández, 2005; Gee, 2014) para reconocer la multiplicidad de acciones que desarrollan los participantes mediante el uso del lenguaje (Austin, 1955). Así mismo, retomamos los postulados de los nuevos estudios de la cultura escrita (New Literacy Studies) que señalan la necesidad de recorrer la escritura directamente en los espacios donde ocurre, para comprender las construcciones ideológicas, históricas y sociales que representa (Street, 2005; Kalman y Street, 2009; Lankshear \& Knobel, 2011).

Construcción de la flexibilidad en una universidad colombiana pública a distancia con modalidad virtual. René Montero y Judith Kalman. 
Este enfoque teórico nos permite analizar el lenguaje escrito en conjunto con los contextos sociales y culturales en que suceden sus usos. En este sentido, consideramos los discursos como sistemas de significados socialmente constituidos los cuales están inmersos en contextos sociales, históricos e institucionales particulares (Gee, 2005; Blommaert, 2005; Hardman, 2008), de modo que nos permiten describir y analizar las formas en que los estudiantes y su docente, construyeron la flexibilidad en el aula digital.

Analizar el lenguaje dio lugar a conocer los modos en que estudiantes y maestra hicieron uso de la escritura en el tiempo específico en que interactuaron (Gee, 2005), y desde ahí, identificar las formas en que construyeron el carácter flexible de su curso. Para esto analizamos los discursos de los participantes (Blommaert, 2005; Gee, 2005) y específicamente los turnos conversacionales (Kalman, 1996) que tuvieron en los foros de trabajo. Posteriormente elaboramos descripciones de las interacciones entre estudiantes y maestra, para comprender cómo se vincularon en redes de relaciones (Habib \& Wittek, 2007), que dieron sentido y significado a la flexibilidad en su proceso de formación a distancia.

Desde esta perspectiva, el trabajo que presentamos se concentra específicamente en lo que sucedió en el aula en línea y explora las relaciones que se establecieron entre los estudiantes, la maestra y la institución. Así describimos algunos de los matices que puede tomar en un aula digital una noción como la flexibilidad, entendida como la característica distintiva de la educación a distancia mediada por el uso de tecnologías y la conectividad, que posibilita que los estudiantes puedan acceder en cualquier momento a desarrollar sus tareas en el aula digital (UNESCO, 2002; OCDE, 2015).

\section{La Universidad del estudio}

El trabajo de campo se desarrolló en la única Universidad pública colombiana que ofrece la totalidad de sus programas a distancia mediante la plataforma Moodle. Esta Universidad tiene presencia en gran parte del territorio colombiano y su población estudiantil consta de personas de diversas edades. Para ingresar a los programas de la institución sólo se requiere realizar una prueba general que no tiene carácter eliminatorio; es decir, que el acceso a los programas está asegurado sólo con haber culminado los estudios de educación secundaria, haber realizado la prueba nacional de egreso de la educación básica y secundaria y presentar la prueba de caracterización exigida institucionalmente.

Cuando el estudiante se matricula, se le asigna un usuario de correo electrónico y otro para el acceso a la plataforma virtual donde desarrolla la mayor parte de sus actividades académicas. Sin embargo, existe una proporción de actividades se llevan a cabo de manera presencial en distintas sedes de la universidad o en lugares con los que la universidad tiene convenios. La institución define su modalidad educativa como un programa de e-learning "basada en los escenarios digitales y telemáticos a través de su Campus Virtual" (UNAD, 2011: 175). No caracteriza su oferta educativa como Blearning, más bien asume a las opciones presenciales como un complemento a su propuesta curricular.

Construcción de la flexibilidad en una universidad colombiana pública a distancia con modalidad virtual. René Montero y Judith Kalman. 
El proceso de acompañamiento de los docentes se centra en asesorías que se desarrollan en plataforma, mediante correos electrónicos, foros de trabajo, sesiones de chat o videoconferencias donde se presentan preguntas y respuestas a las dudas. Así mismo, existe la opción de acompañamientos presenciales que ocurren de manera programada en las sedes de la universidad. Estos pueden ser personalizados, para pequeños grupos o a modo de taller, caso en que los estudiantes de un mismo curso se encuentran, mediante el sistema de conferencia en línea, en simultánea en los centros de la universidad cercanos a sus hogares y desarrollan tareas orientadas por el docente del curso desde la sede en la que dicho docente trabaja. A esta forma de taller se le denomina encuentro B-learning.

En esta institución los docentes tienen dos denominaciones. Por una parte, el director de curso quien, además de tener asignados estudiantes, se encarga de hacer el diseño de las actividades, la organización y el montaje del curso en la plataforma y atender las inquietudes que tengan los estudiantes, relacionadas con las fechas de las actividades y el desarrollo de las prácticas. Adicionalmente, el director de curso se ocupa de asuntos como la apertura y cierre de actividades o la resolución de dificultades con el desarrollo de evaluaciones.

El segundo tipo de docente es el tutor. Este docente se encuentra en los cursos donde el número de estudiantes excede la carga máxima permitida para el director. El tutor se encarga de la atención a los estudiantes, de operar las actividades del curso, hacer acompañamiento a los alumnos, canalizar las dudas que requieran intervención del director y entregar las calificaciones y retroalimentaciones de los trabajos de los estudiantes a su cargo.

\section{El trabajo de campo}

El curso Biología Ambiental contó con un total de tres docentes - una directora y dos tutoras-y 754 estudiantes matriculados, los cuales fueron organizados de manera aleatoria por la plataforma, en 154 grupos de cuatro o cinco. Estos grupos, se asignaron entre las tres docentes quienes tuvieron a su cargo 66 (aprox. 330 estudiantes), 48 (aprox. 240 estudiantes) y 40 (aprox. 200 estudiantes) respectivamente.

El curso constaba de tres unidades temáticas: (1) Introducción a la Biología, Estructura y tipos celulares, Niveles de organización, reinos y dominios; (2) La Biología como elemento del medio ambiente y como elemento para su recuperación y cuidado y (3) Comunicación científica desde el trabajo tanto de campo como de laboratorio.

Para desarrollar las actividades de estas unidades, los estudiantes tuvieron un total de 16 semanas, en las cuales tenían que resolver las tareas asignadas y un examen final de curso. En la Tabla 1, se describen las actividades de cada unidad temática:

Tabla 1. Tareas asignadas en cada una de las unidades del curso.

\begin{tabular}{|l|l|}
\hline \multicolumn{1}{|c|}{ Unidad } & \multicolumn{1}{c|}{ Tareas asignadas } \\
\hline "Introducción a la & $\begin{array}{l}\text { Realizar el dibujo a mano alzada (NO presentar fotos, } \\
\text { Biología, Estructura } \\
\text { tipos celulares, Niveles de o bocetos tomados de internet), de una célula } \\
\text { procariota y una célula eucariota, donde se indique las }\end{array}$ \\
\hline
\end{tabular}

Construcción de la flexibilidad en una universidad colombiana pública a distancia con modalidad virtual. René Montero y Judith Kalman. 


\begin{tabular}{|c|c|}
\hline $\begin{array}{l}\text { organización, reinos } \\
\text { dominios" }\end{array}$ & $\begin{array}{l}\text { estructuras, organelos y procesos o funciones de cada } \\
\text { una. } \\
\text { Hacer un cuadro comparativo de los reinos de la } \\
\text { naturaleza, que incluya las características en cuanto a } \\
\text { estructura, procesos, hábitat y tipo de reproducción. } \\
\text { Hacer un cuadro comparativo de los dominios de los } \\
\text { seres vivos, que incluya las características en cuanto a } \\
\text { estructura, composición y metabolismo } \\
\text { Resolver un estudio de caso }\end{array}$ \\
\hline $\begin{array}{l}\text { "La Biología } r \\
\text { elemento del medio } \\
\text { ambiente y como elemento } \\
\text { para su recuperación y } \\
\text { cuidado" }\end{array}$ & $\begin{array}{l}\text { Realizar un cuadro comparativo que incluya los } \\
\text { elementos que permiten definir un sistema abierto y un } \\
\text { sistema cerrado dando ejemplos concretos y reales de } \\
\text { cada uno de estos sistemas } \\
\text { Determinar si los seres vivos corresponden a sistemas } \\
\text { abiertos o cerrados } \\
\text { Realizar un esquema que muestre el funcionamiento } \\
\text { termodinámico de un ser vivo acompañado de un breve } \\
\text { texto explicativo que lo sustente. } \\
\text { Realice un cuadro comparativo de los tres modelos para } \\
\text { explicar la forma en que ocurre el reemplazo de especies } \\
\text { en una sucesión ecológica. } \\
\text { Explicar por medio de un breve texto la Hipótesis Gaia. }\end{array}$ \\
\hline $\begin{array}{l}\text { "Comunicación científica } \\
\text { desde el trabajo tanto de } \\
\text { campo como } \\
\text { laboratorio" }\end{array}$ & $\begin{array}{l}\text { Realizar un diseño de permacultura eficiente para un } \\
\text { sistema ecológico en Colombia. }\end{array}$ \\
\hline
\end{tabular}

En la primera unidad denominada "Introducción a la Biología, Estructura y tipos celulares, Niveles de organización, reinos y dominios" los estudiantes tuvieron 21 días para desarrollar de manera colaborativa ${ }^{1}$ en el foro, cuatro tareas que incluían dos dibujos de células con sus partes, una tabla que mostrara la clasificación de la naturaleza por dominios, una tabla que mostrara la clasificación de la naturaleza por reinos, y la respuesta a un análisis de caso.

En la segunda unidad: "La Biología como elemento del medio ambiente y como elemento para su recuperación y cuidado", los estudiantes tuvieron 31 días para desarrollar, también de manera colaborativa y en el foro, cuatro tareas que incluían un cuadro comparativo entre un sistema cerrado y uno abierto, un esquema de funcionamiento termodinámico de un ser vivo, un cuadro comparativo entre tres

\footnotetext{
${ }^{1}$ La expresión colaborativo obedece a la forma como se denomina institucionalmente a los trabajos que los estudiantes deben hacer en los foros.
}

Construcción de la flexibilidad en una universidad colombiana pública a distancia con modalidad virtual. René Montero y Judith Kalman. 
modelos de reemplazo de especies en una sucesión ecológica y un ensayo sobre la hipótesis Gaia.

En la tercera y última unidad denominada "Comunicación científica desde el trabajo tanto de campo como de laboratorio", los estudiantes tuvieron 45 días para construir de manera colaborativa y en el foro, un diseño de permacultura eficiente para un sistema ecológico en Colombia.

Los datos de este estudio se tomaron directamente del registro que guarda la plataforma Moodle de esta institución, de las interacciones entre los participantes durante el desarrollo de las tareas de la unidad tres en el foro en línea.

En un lapso de dos meses luego de la finalización del curso, recuperamos los foros de trabajo de los 154 grupos de estudiantes, registramos todos los mensajes de correo enviados y recibidos durante el periodo analizado, las retroalimentaciones que elaboraron las maestras frente a las tareas de cada unidad y los productos finales y parciales que los estudiantes entregaron.

Para este estudio, se seleccionaron los mensajes que los estudiantes y su maestra enviaron a los foros, dado que en los demás espacios como wikis o sistemas de mensajería no se encontraron interacciones conducentes al desarrollo de actividades del curso. Más bien allí se centraron en tratar aspectos administrativos como las fechas de apertura y cierre de las actividades.

Los registros se almacenaron tanto en formato ".pdf", descargado de la plataforma, como en formato ".doc" habilitado para edición. En todos los documentos y mensajes de los participantes se respetaron los formatos y escritura originales, sin alterar aspectos ortográficos o de redacción; también se asignó un pseudónimo para cada uno de los alumnos y la maestra por motivos de confidencialidad.

Describimos estas producciones y estos discursos con el fin de comprender y analizar las formas en que se construye la flexibilidad en el caso específico del curso del estudio. Dado que todos los datos provienen de los mensajes que escribieron los participantes, analizamos los usos del lenguaje (Blommaert, 2005), lo cual abre la posibilidad de examinar las formas en que sucede la construcción de relaciones y significados específicos en los intercambios (Gee, 2005).

\section{La sistematización de los datos}

A pesar de que el levantamiento de datos en espacios digitales no permita reconocer la totalidad de prácticas que desarrollan los usuarios (Hine, 2004:70), sí es posible hacer un acercamiento a algunas situaciones puntuales a partir del análisis de las interacciones que realizaron los participantes del aula en línea. En este caso nos aproximamos a las formas en que los estudiantes y docentes realizaron actividades académicas y, en el proceso de comunicarse, intercambiar archivos y llegar a acuerdos, dieron forma a la flexibilidad del tiempo, en sus cursos virtuales.

Buscamos conocer, desde la evidencia empírica, los significados sobre el uso flexible del tiempo que construyeron los participantes (Heath, 1982, Castanheira, 2001, Green, Dixon \& Zaharlick, 2005), y ofrecer resultados descriptivos, más allá de pretender la

Construcción de la flexibilidad en una universidad colombiana pública a distancia con modalidad virtual. René Montero y Judith Kalman. 
formulación de juicios sobre las formas de participación que tuvieron los estudiantes y la maestra en el aula digital (Hine, 2004).

En esta investigación, el análisis del discurso se constituyó en una perspectiva teóricometodológica desde la cual los discursos de los participantes, representados en los textos que construyeron y compartieron con sus compañeros en el foro, se entendieron como sistemas de significados socialmente constituidos e inmersos en contextos sociales, históricos e institucionales particulares (Hardman, 2008).

Para analizar el discurso de los estudiantes, se tomaron cada uno de los turnos (Kalman, 1996) registrados en los foros de trabajo y se ubicaron en una hoja de cálculo manteniendo los datos de fecha y hora en que fueron publicados. Para este fin se siguió la propuesta de Kalman y Rendon (2016), por considerar que el trabajar con datos cualitativos en esta forma, permite rastrear información específica y registros particulares mediante el diseño específico de la forma en que se quieren analizar los aspectos que se van a describir.

Cada intervención de un participante - docente o estudiante - en el foro, fue separada y numerada por líneas (Gee, 2005) y se conservaron tanto la escritura original del registro como el orden cronológico en que había sucedido. Esto significa que hemos reproducido fielmente los mensajes de los participantes, respetando su ortografía, sintaxis y fraseo. Los números de línea que se emplearon en cada fragmento corresponden a aquellos con los que fueron codificados en la transcripción original.

El trabajo consistió en identificar en cada turno las oraciones o fragmentos de texto que daban cuenta de una acción particular (Gee, 2005), para luego ubicarlas por líneas separadas. Esta organización, no desarticuló los textos de los participantes, más bien permitió reconocer las acciones que ellos llevaron a cabo mediante el uso e intencionalidad del lenguaje (Austin, 1955), para analizarlas de manera específica en el contexto en el que fueron producidas.

Como se muestra en la Tabla 2., para cada fragmento en los turnos, se construyó un descriptor (expresado en un verbo conjugado más un complemento), que representaba lo que estudiantes y docentes estaban haciendo mediante el uso del lenguaje (Kalman y Rendón, 2016).

Tabla 2. Organización de datos del turno de la maestra en la hoja de cálculo

\begin{tabular}{|c|c|c|c|l|l|}
\hline Fecha & Hora & Participante & Línea & \multicolumn{1}{|c|}{ Turno } & \multicolumn{1}{|c|}{ Descriptor } \\
\hline $\begin{array}{c}2 \text { de } \\
\text { mayo }\end{array}$ & $14: 45$ & Maestra & 43 & $\begin{array}{l}\text { Estimado Hernando, } \\
\text { Buenas tardes, }\end{array}$ & MarcaReceptores \\
\hline $\begin{array}{c}2 \text { de } \\
\text { mayo }\end{array}$ & $14: 45$ & Maestra & 44 & $\begin{array}{l}\text { aun queda tiempo } \\
\text { para que subas tus } \\
\text { aportes }\end{array}$ & $\begin{array}{l}\text { IndicaTiempo } \\
\text { (Flexibilidad) }\end{array}$ \\
\hline $\begin{array}{c}2 \text { de } \\
\text { mayo }\end{array}$ & $14: 45$ & Maestra & 45 & $\begin{array}{l}\text { por favor cordina con } \\
\text { tus compañeros para } \\
\text { que todos trabajen } \\
\text { sobre el mismo } \\
\text { sistema Ecologico }\end{array}$ & AsignaTarea \\
\hline $\begin{array}{c}2 \text { de } \\
\text { mayo }\end{array}$ & $14: 45$ & Maestra & 46 & $\begin{array}{l}\text { Cordialmente Liliana } \\
\text { CierraTurno }\end{array}$ & \\
\hline
\end{tabular}

Construcción de la flexibilidad en una universidad colombiana pública a distancia con modalidad virtual. René Montero y Judith Kalman. 


\begin{tabular}{|c|c|c|c|l|l|}
\hline $\begin{array}{c}3 \text { de } \\
\text { mayo }\end{array}$ & $10: 24$ & Daniel & 47 & Cordial saludo & AbreTurno \\
\hline $\begin{array}{c}3 \text { de } \\
\text { mayo }\end{array}$ & $10: 24$ & Daniel & 48 & $\begin{array}{l}\text { estimada tutora y } \\
\text { compañeros. }\end{array}$ & MarcaReceptores \\
\hline $\begin{array}{c}3 \text { de } \\
\text { mayo }\end{array}$ & $10: 24$ & Daniel & 49 & $\begin{array}{l}\text { Hago entrega de mi } \\
\text { aporte } \\
\text { correspondiente al } \\
\text { cuadro de } \\
\text { permancultura }\end{array}$ & AnunciaEntrega \\
\hline $\begin{array}{c}3 \text { de } \\
\text { mayo }\end{array}$ & $10: 24$ & Daniel & 50 & $\begin{array}{l}\text { el cual me base en una } \\
\text { reserva natural como } \\
\text { lo es la laguna de la } \\
\text { cocha un lugar muy } \\
\text { bello en el cual } \\
\text { predominan muchas } \\
\text { especies y muchas } \\
\text { afluentes } \\
\text { hidrografícas, }\end{array}$ & DescribeEntrega \\
\hline $\begin{array}{c}3 \text { de } \\
\text { mayo }\end{array}$ & $10: 24$ & Daniel & 51 & $\begin{array}{l}\text { espero cumpla con lo } \\
\text { requerido, }\end{array}$ & SolicitaComentario \\
\hline $\begin{array}{c}3 \text { de } \\
\text { mayo }\end{array}$ & $10: 24$ & Daniel & 52 & $\begin{array}{l}\text { Gracias por las } \\
\text { atenciones mostradas. } \\
\text { Daniel }\end{array}$ & CierraTurno \\
\hline
\end{tabular}

Este trabajo permitió reconocer que los estudiantes y la maestra realizaron varias acciones en sus turnos. Hicieron uso de aspectos formales, como abrir y el cerrar sus mensajes con saludos o agradecimientos (líneas 46, 47 y 52), o marcar el destinatario de sus mensajes (líneas 43 y 48). En cuanto a las acciones que convencionalmente desarrollan maestros o estudiantes, la maestra se encargó de la asignación de responsabilidades (línea 45) y los estudiantes, por su parte se ocuparon de la realización y entrega de las tareas que se le asignaron (línea 49).

Como podrá verse en el siguiente apartado, fue posible identificar las formas en que los participantes hicieron uso del tiempo, como sucedió en la línea 44 de la tabla 2; y con base en la organización y agrupación de los descriptores, pudimos identificar el carácter flexible como una categoría de análisis, que aparecía de manera reiterada en las interacciones de los participantes.

\section{Cuando la flexibilidad llega al aula en línea}

Para la tercera unidad del curso denominada "Comunicación científica desde el trabajo tanto de campo como de laboratorio", cada grupo de estudiantes contaba con 45 días para construir de manera colaborativa en el foro un diseño de permacultura eficiente para un sistema ecológico en Colombia.

De acuerdo con las indicaciones registradas en la guía de actividades, la maestra esperaba que los estudiantes desarrollaran las tareas primero de manera individual, y luego que las socializaran en el foro para contar con las opiniones de sus compañeros. A

Construcción de la flexibilidad en una universidad colombiana pública a distancia con modalidad virtual. René Montero y Judith Kalman. 
partir de compartir y comentar los trabajos individuales, los alumnos tenían que construir entre todos y en el foro un documento para entregar. El producto final debería ser elaborado a partir de la discusión sobre los trabajos individuales previamente compartidos.

En el grupo de donde se tomaron los datos que a continuación se presentan, los participantes fueron la maestra Liliana y los estudiantes Julián, Hernando, Daniel, Juan y Vicente. Como se muestra en la figura 1, los intercambios entre los estudiantes iniciaron el día 31 y ocuparon 13 de los 45 días disponibles para completar y entregar el trabajo a la maestra. Julián intervino en 7 oportunidades, Hernando en 9, Daniel en 5, Juan en 1 y Vicente no participó en este trabajo.

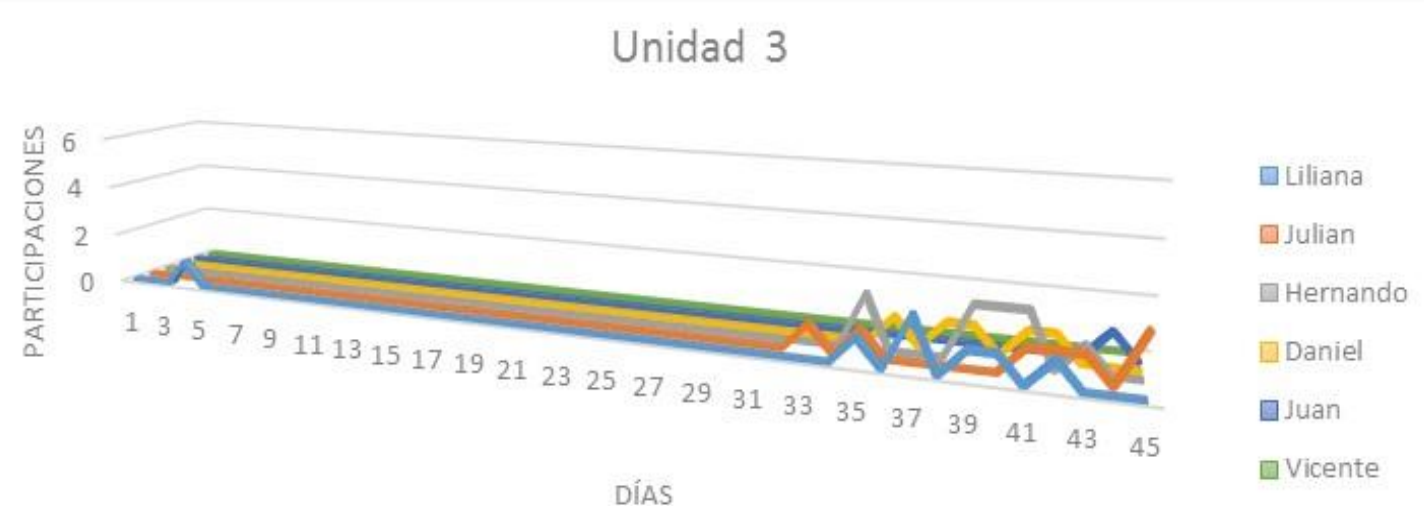

Figura 1. Días en los que ocurrieron los turnos de la maestra y los estudiantes en la unidad 3.

Aunque las interacciones entre los cuatro estudiantes que participaron sucedieron dentro de los 45 días programados por la maestra, uno de ellos, Juan, no apareció como autor en el trabajo final.

Juan entregó su tarea el día 44, es decir, un día antes de la fecha límite para enviar a la maestra. Compartió con sus compañeros en el foro un documento que contenía la gráfica con el diseño de permacultura solicitado por la profesora, la explicación de dicha gráfica y la ejemplificación en un contexto real del diseño de permacultura presentado. No obstante, sus compañeros, específicamente Julián, optaron por no incluir sus aportes dentro del trabajo que enviaron como grupo.

Este ejemplo permite ilustrar cómo la flexibilidad en el manejo del tiempo puede tomar diferentes matices en un aula digital. El primero es que aun cuando se promueve en los discursos oficiales que los estudiantes pueden ingresar a la plataforma a desarrollar sus actividades sin límites de tiempo, la política académica de la Universidad define que cuentan con 45 de los casi 130 días que dura el periodo académico para desarrollar las tareas de la unidad 3. Es decir, hay límites institucionales.

Esto significa que lo flexible, entendido como la posibilidad que tiene el estudiante para decidir sobre los lapsos que dispone para desarrollar y entregar sus tareas en el aula en línea, opera dentro de los límites que impone la institución y de las condiciones que se definen entre compañeros, pero no se expresan, en cuanto a los momentos en que sucederán las interacciones para desarrollar las tareas asignadas.

Construcción de la flexibilidad en una universidad colombiana pública a distancia con modalidad virtual. René Montero y Judith Kalman. 
El segundo matiz de esta flexibilidad consiste en que al promover cierta libertad en el manejo del tiempo para el acceso al aula y para el desarrollo de las tareas, los estudiantes ocupan mayormente días de descanso (días festivos o fines de semana) y horarios fuera de la jornada laboral (nocturnos o temprano en las mañanas) para ingresar y dejar sus aportes, como se muestra a continuación.

\section{de Hernando-jueves 30 de abril, 23:57}

27 Cordial saludo Tutora;

28 Buenas noches apreciada Tutora Liliana,

29 de la manera mas formal me dirijo a usted con el fin de disculparme ya que no e podido realizar mis aportes

30 ya estoy trabajando para en la semana estar haciendo llegar mis aportes.

31 Ademas muchas gracias por estar pendiente de los aportes realizados por los compañeros.

32 Cordialmente; Hernando

Este turno es el primero de Hernando en la unidad y sucedió sobre la media noche, 33 días después del inicio del periodo establecido para el desarrollo de la actividad. El estudiante aprovechó su mensaje para realizar varias acciones. Marcó a la maestra como destinataria (líneas 27 y 28), presentó una disculpa por la demora en el inicio de sus intervenciones (línea 29), anunció el próximo envío de sus aportes (línea 30) y reconoció que el desarrollo de las tareas que conforman la actividad de la unidad ya había iniciado (línea 31) por cuanto 2 días antes de su turno, su compañero Julián ya había intervenido en el foro y había enviado un documento con una entrega parcial.

La maestra respondió el mensaje de Hernando en el foro 2 días después de que el estudiante lo escribiera.

\section{de Liliana-sábado 2 de mayo, 14:45}

43 Estimado Hernando, buenas tardes

44 Buenas tardes, aun queda tiempo para que subas tus aportes

45 por favor cordina con tus compañeros para que todos trabajen sobre el mismo sistema Ecologico

46 Cordialmente, Liliana

En su turno, la maestra realizó dos acciones principalmente. En primer lugar, puso en escena el carácter flexible del curso al señalar al estudiante que tenía tiempo para realizar su trabajo e interactuar con los demás (línea 44), esto porque aún restaban ocho días para hacer la entrega final del documento solicitado; y en segundo lugar asignó a Hernando la responsabilidad de coordinar con sus compañeros el trabajo y asegurar que todos se enfocaran en el mismo ecosistema (línea 45).

Ese turno muestra dos aspectos relevantes. En primer lugar, las acciones de maestros y estudiantes, por lo menos en los datos analizados, no resultaron ser sustancialmente diferentes de lo que puede encontrarse entornos presenciales. En este caso, la maestra le asignó al estudiante la responsabilidad de coordinar la elección del ecosistema con que trabajaría el equipo.

Construcción de la flexibilidad en una universidad colombiana pública a distancia con modalidad virtual. René Montero y Judith Kalman. 
En segundo lugar, se puede ver una consecuencia de que la participación de los estudiantes en los foros suceda de manera asincrónica (Nandi, Hamilton \& Harland, 2012). Cuando un alumno tiene una pregunta o solicitud, esta no se resuelva de manera inmediata, tiene que esperar a que la persona a quien se dirigió ingrese y la responda (Andresen, 2009). En este caso, la maestra tomó dos días para contestar a Hernando.

El caso de Daniel fue similar al de Hernando:

\section{de Daniel-viernes 1 de mayo, 12:40}

33 Cordial saludo estimada tutora y compañeros.

34 Revisando el foro

35 me gustaría felicitar al compañero Julián

36 ya que es muy interesante el cuadro realizado por el compañero,

37 ademas ofrezco excusas por la demora en mis aportes

38 estaré trabajando en ellos.

39 Gracias por las atenciones mostradas.

40 Daniel

En su turno señaló como destinatarios del mensaje a la maestra y a sus compañeros (línea 33), anunció que había estado pendiente del trabajo realizado hasta el momento (línea 34), sugirió que había revisado los aportes y emitió un juicio sobre ellos (línea 36), presentó una disculpa por la demora en el inicio de sus aportes (línea 37) y anunció que ya había iniciado con su trabajo (línea 38).

La respuesta a su mensaje llegó 3 días después.

\section{de Liliana-lunes 4 de mayo, 07:26}

41 Estimado Daniel, Cordial saludo,

42 recuerda que debes incluir un texto explicativo de la manera como se a va desarrollar cada componente de tu modelo

43 Cordialmente Liliana

Al igual que en el caso de Hernando, la maestra empleó su turno para asignar tareas (línea 42), pero en este caso particular además de la asignación de la tarea, la maestra reiteró el trabajo que debía hacer el estudiante y que estaba escrito en la guía de actividades.

Un aspecto que tienen en común Daniel y Hernando es el hecho de haber empleado sus turnos para realizar acciones que no tienen contenido académico explícito. Anunciaron entregas de documentos y se manifestaron su acuerdo con el trabajo de los demás, pero sin discutir sobre los contenidos de biología.

Esto quiere decir que las discusiones académicas posiblemente se llevaron a cabo en momentos y espacios diferentes a los que se ocuparon en permanecer en la plataforma. De modo que, por lo menos en el caso analizado, el foro se destinó al envío de documentos ya elaborados y a la formulación de comentarios formales (línea 35) sobre el trabajo de los demás.

Otro aspecto que resalta de los turnos iniciales de Hernando y Daniel tiene que ver con las horas del día y los días en que enviaron mensajes al foro. Hernando inició su trabajo

Construcción de la flexibilidad en una universidad colombiana pública a distancia con modalidad virtual. René Montero y Judith Kalman. 
sobre la media noche y Daniel lo hizo un día festivo oficial en Colombia. Estas situaciones son una muestra de cómo se viven las consignas que promueven los organismos oficiales, y la institución del estudio, sobre la flexibilidad en el manejo del tiempo (UNESCO, 2002; UNAD, 2011; OCDE, 2015), entendida como la posibilidad de acceder a las plataformas a desarrollar las actividades durante 24 horas del día, cualquier día.

Sin embargo, los estudiantes no inician necesariamente sus participaciones en los cursos desde el momento mismo en que se abren las actividades. El que Hernando y Daniel usaran sus turnos para presentar disculpas por la demora que tuvieron en comenzar sus intercambios en el foro, no significa que Julián, el compañero que participó primero, lo hubiera hecho el día 1 o 2 de los 45 proyectados para la unidad; él esperó cuatro semanas para hacer su primera intervención en el foro.

\section{de Julián-martes 28 de abril, 21:32}

5 Buenas noches tutora y compañeros

6 Junto con saludar adjunto el sistema de permacultura

7 segun guía

8 Espero comentarios de la tutora

9 para corregir si es necesario

10 Gracias

En su turno dirigido a la maestra y a sus compañeros (línea 5), Julián hizo una entrega parcial del trabajo de la unidad (línea 6), y pidió que la maestra lo revisara (línea 8). Esta solicitud del estudiante muestra su mirada sobre la forma que toma la colaboración en las tareas a realizar. Si bien para la maestra debe conseguirse un producto con la participación de todos, para Julián las revisiones de contenidos sobre las entregas parciales están delegadas en la maestra exclusivamente (línea 8).

De esta manera, desde las mismas prácticas de los participantes, es posible cuestionar discursos que proponen que la articulación de tecnologías, políticas institucionales y propuestas educativas en los espacios digitales de formación potencian la autonomía de los estudiantes en la gestión de sus propios procesos académicos (Moodle, 2015; Novo, 2004; Ruiz, 2016; Bejarano y González, 2017) y promueven cambios de roles entre docentes y estudiantes (Dávila, Ruíz y Francisco, 2013; Moodle, 2015; Silva, 2017). Los datos muestran que las experiencias previas de maestros y alumnos, así como sus expectativas e ideas en torno a lo que significa hacer escuela-por ejemplo, el estudiante hace y el profesor revisa-, juegan un papel central en las interacciones que sostienen en sus cursos digitales.

Hasta este momento, los tiempos de participación de los estudiantes han sucedido de acuerdo con lo que se formula por parte de las agencias internacionales y de la política institucional. Es decir que los estudiantes han ingresado al foro de trabajo en los tiempos que han tenido disponibles para hacerlo, y dentro de los lapsos definidos por la Universidad.

Sin embargo, aunque los alumnos han desarrollado sus trabajos de manera individual, la colaboración en la actividad no sucedió de acuerdo con las orientaciones de la profesora. Su expectativa, planteada explícitamente en la guía de actividades, era que los alumnos hicieran las tareas primero de manera individual, posteriormente las

Construcción de la flexibilidad en una universidad colombiana pública a distancia con modalidad virtual. René Montero y Judith Kalman. 
socializaran y discutieran en el foro, y luego colaboraran para construir un único documento grupal. Aunque los estudiantes tuvieron intercambios en el foro, fueron escasos los comentarios, preguntas y discusiones sobre el contenido disciplinar y sobre las estrategias para mejorar los aspectos formales de los textos; es decir, las interacciones académicas alrededor de sus escritos en el foro fueron mínimas. No obstante, es posible que algunos de ellos hayan sostenido intercambios por medio de redes sociales o llamadas telefónicas; sin embargo, no se recopilaron este tipo de datos $\mathrm{y}$ en los registros del foro no hay evidencia de que esto haya ocurrido.

Este es otro matiz de la flexibilidad en educación a distancia. El contar con la posibilidad de acceder al foro para construir el desarrollo de las tareas en cualquier momento, tiene implicaciones en el desarrollo del trabajo en grupo, las cuales no están registradas ni descritas en las consignas oficiales o institucionales.

Al parecer, el hecho de realizar un trabajo bajo las indicaciones de colaboración que solicita la maestra implicaría cuando menos dos condiciones. Por una parte, el contar con espacios adicionales al foro, como chats, para el encuentro sincrónico, no necesariamente presencial, donde los estudiantes puedan comentar sus trabajos sin esperar lapsos amplios a obtener respuestas o retroalimentaciones de sus maestros y compañeros (Andresen, 2009). Por otra parte, un acompañamiento intencionado de parte del docente, más sólido y permanente, donde se construyan estas formas de trabajo con los alumnos para que en el curso y a futuro, puedan organizarse con mayor facilidad, tomar decisiones y resolver las situaciones que se presenten sobre las formas de interacción del grupo para elaborar los productos a entregar.

Pasados nueve días de la primera intervención de Julián, es decir 3 días antes de la fecha límite, se perfila la definición del documento a entregar a la maestra.

\section{de Julián- jueves 7 de mayo, 08:27}

119 Buenos dias compañeros

120 Yo propongo realizar como trabajo final, mi trabajo

121 ta que en la guia solo se exige el grafico y el detalle mas la portada de loas que colaboraron.

122 Asi que se los dejo para que durante este dia lo discutamos.

123 Saludos

Julián propuso que su trabajo se entregara como producto grupal a la maestra (línea 120) y solicitó que sus compañeros discutieran su propuesta (línea 122). Aun cuando faltaban tres días para que se cumpliera el tiempo de entrega, y que Juan y Vicente no hubieran intervenido en el foro, Julián solicitó que se definiera ese mismo día la entrega. En su turno llama la atención que sólo se dirige a los compañeros, de modo que la discusión sobre el trabajo a entregar excluyó a la maestra.

A la propuesta de Julián, respondió Daniel pasadas 10 horas.

\section{de Daniel- jueves 7 de mayo, 18:14}

127 Cordial saludo estimada tutora y compañeros.

128 Estimado compañero Julián

129 estoy de acuerdo en lo que usted propone,

130 espero podamos realizar un optimo trabajo final.

Construcción de la flexibilidad en una universidad colombiana pública a distancia con modalidad virtual. René Montero y Judith Kalman. 


\section{Gracias por las atenciones mostradas. Daniel}

La respuesta de Daniel menciona a la maestra como destinataria del mensaje. En el turno previo, Julián no la había incluido, quizá porque la consulta que estaba haciendo sólo era de competencia de los estudiantes. Pero ahora cuando Daniel respondió a esa consulta, volvió a llamar a la docente-lo cual podría leerse como una manera de hacer notar su participación (línea 127), y aprobó la propuesta de Julián (línea 129), sin comentar su contenido o manifestar algún criterio para haber tomado dicha determinación. Sin embargo, durante el día no se registró la opinión de Hernando. Frente a lo cual, al día siguiente Julián nuevamente requirió a Hernando para definir la entrega.

\section{de Julián-viernes, 8 de mayo, 09:39}

132 Buenos dias

133 Daniel y Hernando y por supuesto tutora.

134 Y es dia viernes 8, a 2 dias de entregar el trabajo,

135 yo propuse el mio para entregar,

136 daniel esta de acuerdo,

137 que dices tu Hernando?

138 nesecito que respondas luego en el dia de hoy

139 para consolidar el trabajo final.

140 Saludos

En esta oportunidad el mensaje de Julián incluyó a la maestra (línea 133), en su turno señaló el tiempo que restaba para realizar la entrega (línea 134), recordó que había postulado su trabajo como producto final (línea 135), confirmó que Daniel había aprobado dicha iniciativa (línea 133), requirió la respuesta de Hernando (línea 137) y marcó que ese día esperaba contar con la respuesta de su compañero (línea 138).

El turno de Juan ejemplifica una dificultad que se puede generar entre la propuesta de la maestra, resolver una tarea de forma colaborativa, y el hecho de que los alumnos accedan a los cursos de acuerdo con los tiempos que tengan disponibles. La sugerencia de la docente de que los alumnos sostengan participaciones con discusiones para construir los productos académicos supone una cierta regularidad en la frecuencia de participaciones en el foro, lo cual podría ser una dificultad para quienes tengan limitaciones de tiempo para desarrollar sus estudios. Más aún cuando el carácter flexible que caracteriza la educación a distancia, en este caso, solo funciona dentro de los lapsos que la institución ha definido para elaborar los productos de cada unidad

Así mismo, que un estudiante pueda desarrollar las actividades en cualquier momento está condicionado por las indicaciones de la maestra. En esta unidad, Liliana esperaba que los estudiantes primero desarrollaran las tareas de manera individual, luego las socializaran y comentaran con los demás y, finalmente, sostuvieran discusiones sobre el contenido académico para elaborar un producto colectivo. Esta serie de acciones puede limitar la posibilidad de ingresar en los tiempos que tengan disponibles, como lo señalan las consignas sobre flexibilidad, pues requiere que la presencia de los alumnos en el foro sea permanente para interactuar con sus compañeros.

Construcción de la flexibilidad en una universidad colombiana pública a distancia con modalidad virtual. René Montero y Judith Kalman. 
Contrario a la expectativa de la maestra, en este grupo no hubo discusiones colectivas. El producto final se definió de la siguiente manera, Julián postuló su tarea como trabajo grupal esperando que sus compañeros validaran la propuesta.

Ante el requerimiento de Julián, Hernando contestó dos horas después.

\author{
de Hernando- viernes 8 de mayo, 11:39 \\ 141 Cordial saludo; \\ 142 Buenos dias compañero Julián, \\ 143 el motivo de esta nota es con el fin de felicitarlos a usted y \\ a mi compañero Daniel \\ 144 por su dedicacion y participacion, \\ 145 además si estoy de acuerdo con lo que usted propone \\ 146 ya que su trabajo esta mas bien fundamentado. \\ 147 Gracias y muchos éxitos
}

La aceptación de Hernando frente a la propuesta (línea 145), estuvo acompañada de un comentario frente al trabajo de Julián (línea 146). Al igual que en el caso de Daniel, dicho comentario (línea 146) no incluyó ninguna discusión disciplinar.

Si bien, esta expresión de Hernando sugiere que el estudiante realizó una revisión del documento de su compañero. Sin embargo, los criterios que empleó el alumno para valorar el trabajo de su compañero no fueron visibles en el foro.

Hasta este momento tres de los cinco estudiantes del grupo habían participado y al parecer ya habían definido la entrega final del documento solicitado por la maestra. Pero aún quedaban 2 días de los 45 con que los estudiantes contaban para desarrollar las tareas de la unidad.

Al día siguiente Juan hizo su primera intervención.

\title{
de Juan- sábado 9 de mayo, 19:53
}

148 Buenas noches tutora y compañeros

149 adJunto el trabajo de la actividad 3

150 Espero comentarios

151 Gracias

Juan dirigió su mensaje a la maestra y a los compañeros (línea 148) entregó un documento completo con el diseño de permacultura (línea 149) y les solicitó comentarios (línea 150). La tarea de Juan incluía la descripción de lo que debía tener una finca para ser sustentable, las fuentes de energías renovables a utilizar, la ubicación de la infraestructura, de los productos y subproductos de cultivos agrícolas como tomate y café en variedades orgánicas e inorgánicas. Es decir, el estudiante cumplió con la totalidad de los puntos solicitados por la maestra.

Previo al turno de este estudiante, sus compañeros habían realizado un total de cuatro entregas parciales, dos de Julián, una de Daniel y una de Hernando. Julián entregó dos documentos, uno que no se podía abrir y otro en el que presentaba su gráfica del diseño de permacultura (Figura 2). Además, entregó una descripción donde señalaba que su trabajo era una propuesta para beneficiar el ganado de varias fincas con el agua de una quebrada de la zona.

Construcción de la flexibilidad en una universidad colombiana pública a distancia con modalidad virtual. René Montero y Judith Kalman. 


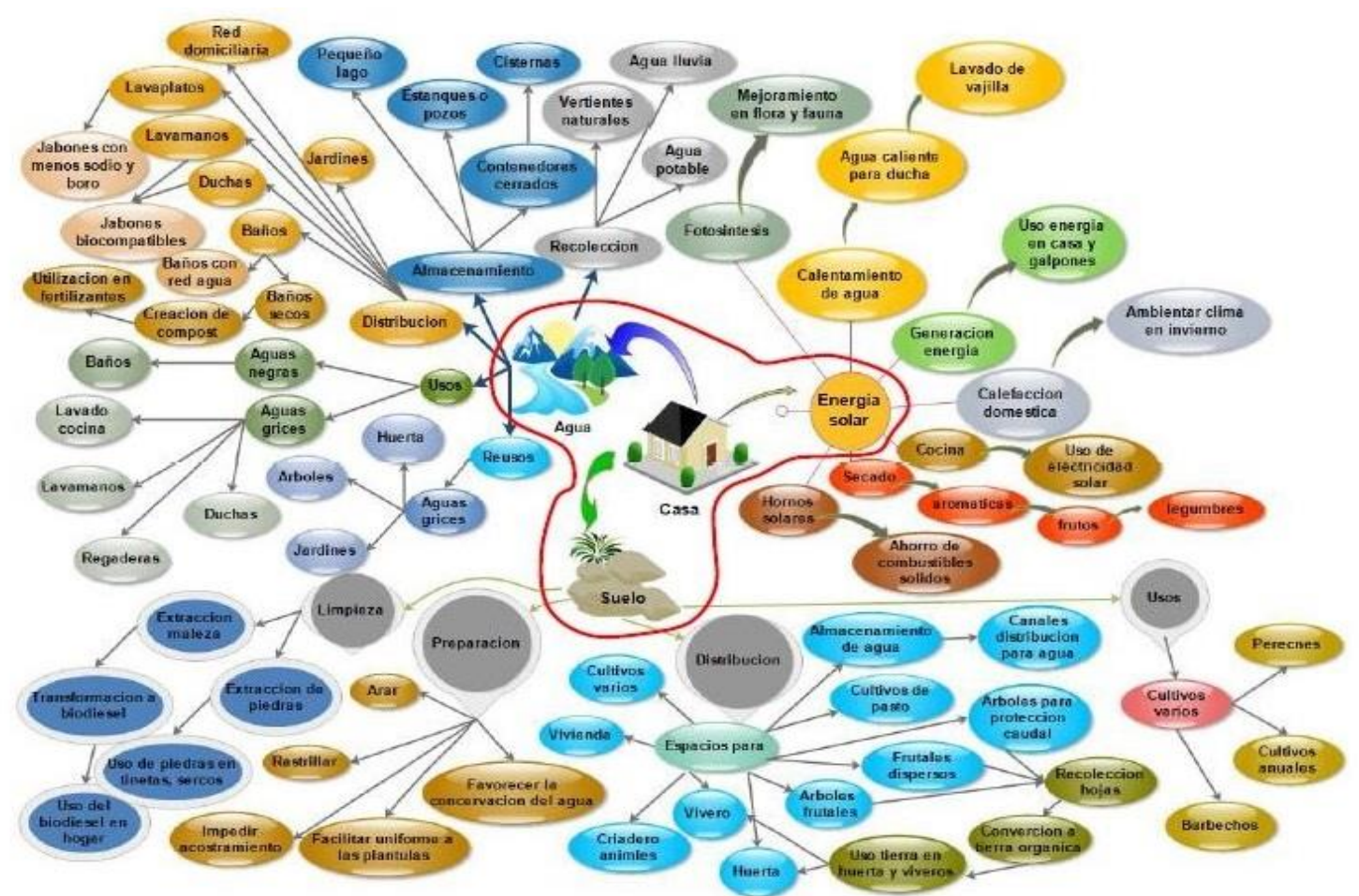

Figura 2. Gráfico de propuesta de permacultura entregado por Julián

Hernando, al igual que Julián, entregó un documento con la gráfica del diseño de permacultura (Figura 3) y la descripción donde mencionaba que su propuesta era la preservación de un páramo a partir de disminuir la intervención humana en el lugar.

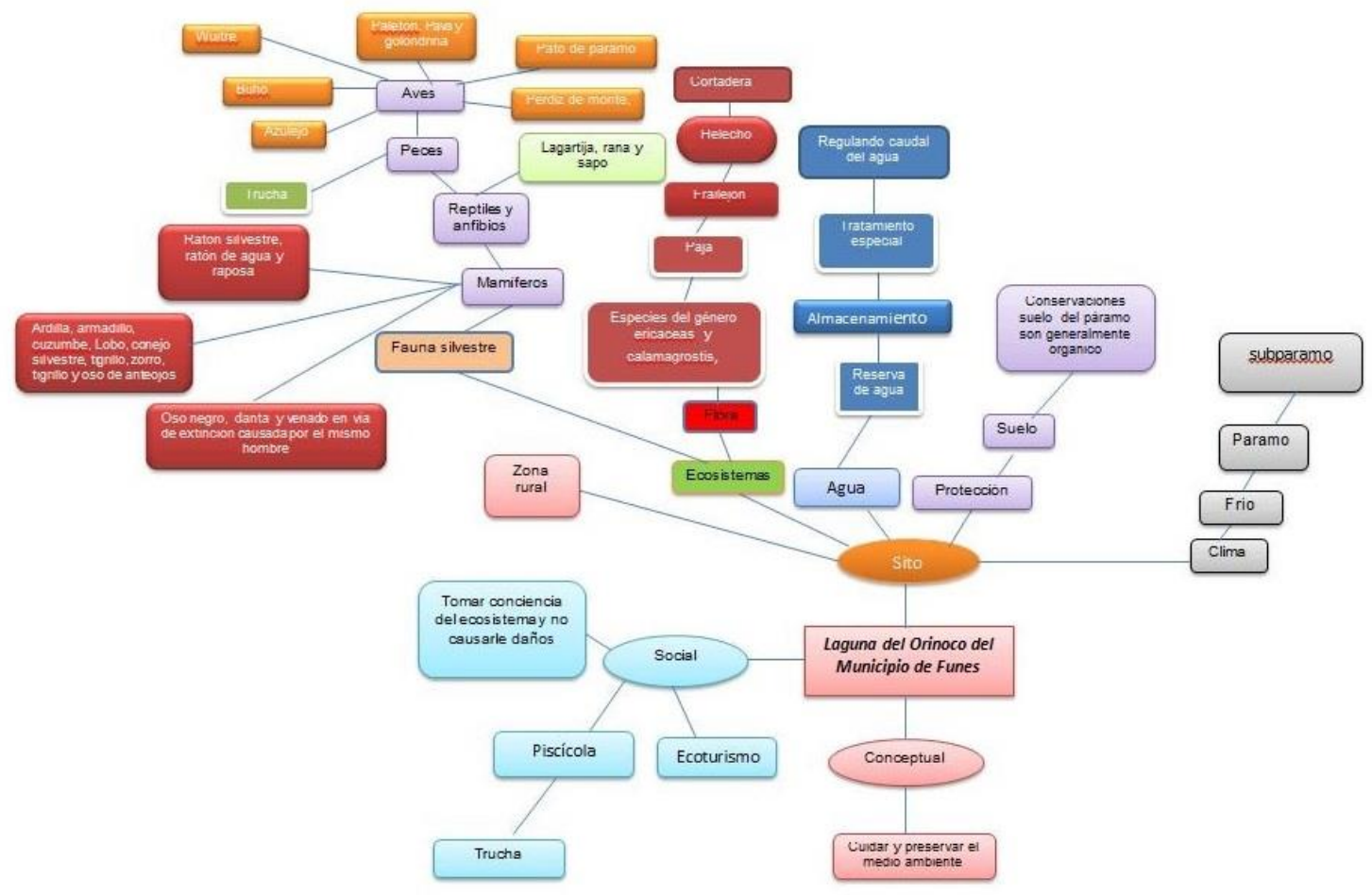


Figura 3. Gráfico de propuesta de permacultura entregado por Hernando

Daniel sólo compartió con sus compañeros la gráfica del diseño de permacultura (Figura 4), que proponía una intervención para proteger la Laguna de la Cocha, la cual se ubica en el departamento de Nariño al sur de Colombia.

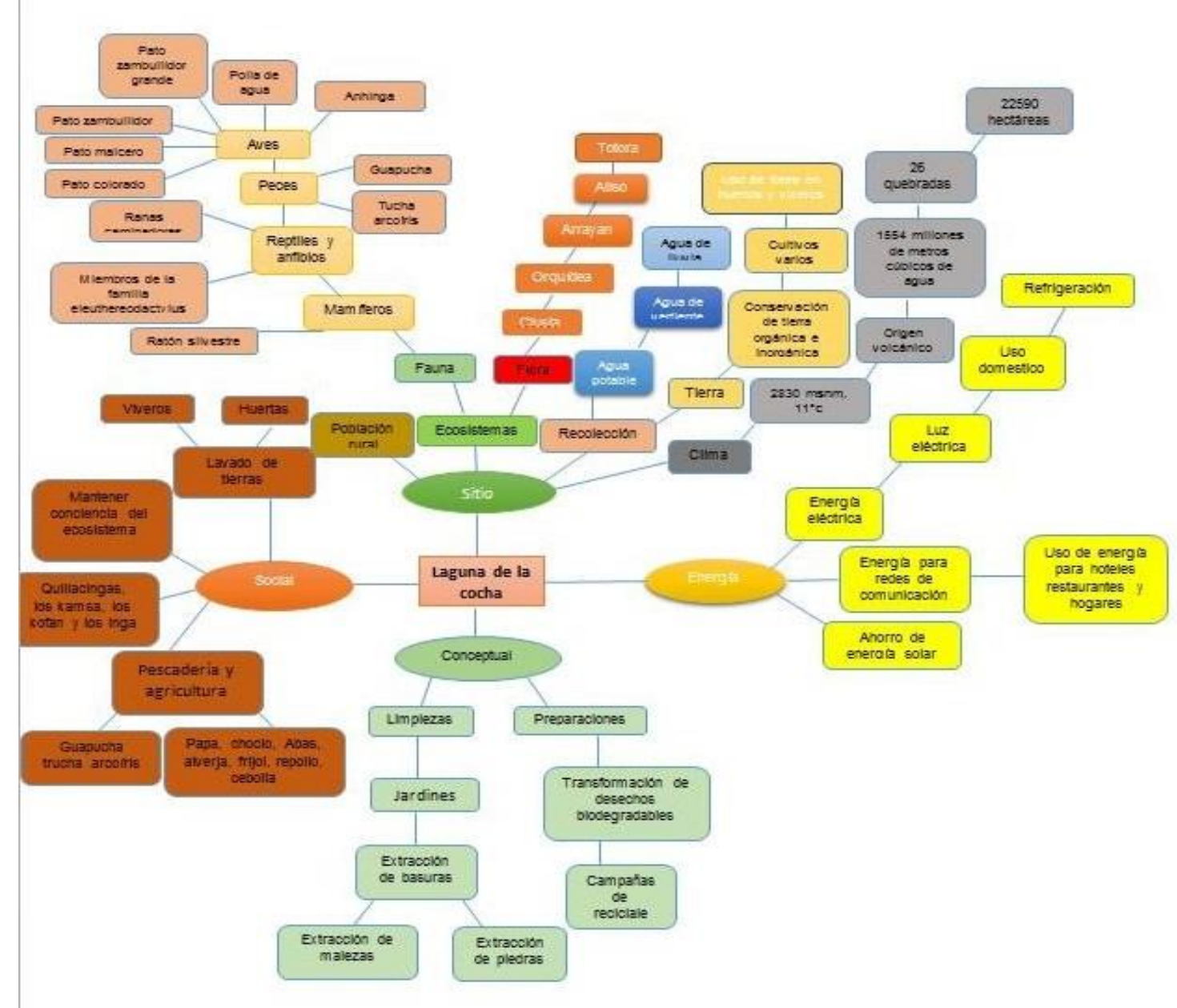

Figura 4. Gráfico de propuesta de permacultura entregado por Daniel

Ninguna de las entregas señaladas tenía la totalidad de aspectos entregados por Juan. A Julián y a Hernando les hacía falta la descripción detallada de la integración de los factores mencionados en su gráfica y a Daniel le hizo falta tanto la explicación del diseño de permacultura como la descripción detallada de la integración de los factores en su propuesta.

Juan solicitó a sus compañeros que hicieran comentarios frente al trabajo que había entregado, pero no obtuvo ningún comentario de la maestra o de los demás integrantes del grupo. 
Aunque los alumnos entregaron sus trabajos dentro del tiempo habilitado institucionalmente, Julián, un día después de la entrega de Juan, desconoció este trabajo y entregó el documento final con sus aportes, los de Hernando y los de Daniel.

\section{de Julián- domingo 10 de mayo, 15:00}

152 Buenas tardes compañeros

153 Subo el trabajo final ya listo

154 Saludos

En este caso, la flexibilidad en términos del manejo del tiempo para realizar y entregar los trabajos de acuerdo con la disponibilidad de los participantes (Jaggars, 2014), parece haber tenido otro matiz en el grupo.

Ninguno de los participantes emitió algún comentario sobre el trabajo de Juan, o se manifestó respecto de que aun hubiera tiempo para revisar el aporte y considerarlo dentro del trabajo del grupo, aun cuando su turno se hubiera realizado dentro de los tiempos dispuestos por la maestra.

Julián desconoció el trabajo de Juan y se otorgó el derecho de definir en qué momento se debía entregar el documento final (línea 134), sin que esto implicara algún comentario de la maestra o de los compañeros.

Juan entregó sus tareas dentro del tiempo establecido institucionalmente sin dificultades, gracias a que el foro de trabajo estuvo abierto desde las 00:05 horas del día 1-que marca el inicio de la unidad 3-, hasta las 23:55 horas del día 45-que marca el final de la misma unidad. Sin embargo, haber enviado su tarea, aún dentro de los límites de tiempo establecidos institucionalmente, no garantizó que los demás integrantes de su grupo aceptaran el envío de su aporte en el documento final entregado a la maestra.

Al parecer, la flexibilidad en términos del tiempo en que se puede acceder a realizar las actividades en línea, promovida desde el discurso de las agencias internacionales y la institución educativa, requiere de acompañamiento y formación a los estudiantes para puedan manejar las diferencias de tiempos para estudiar, y construir formas de organización que les permitan trabajar en equipo.

Cuando los alumnos deben realizar actividades grupales en espacios como el foro en línea, interactúan mayormente de manera asincrónica (Croxton, 2014) y se organizan sobre acuerdos que no se expresan, pero que reflejan sus ideas y expectativas a cerca de lo que es realizar tareas en equipo. En consecuencia, la posibilidad de trabajar en grupo depende en gran medida de la disponibilidad que tengan los estudiantes para atender sus actividades académicas, y de que a los maestros se les asigne el tiempo suficiente para orientarlos en este sentido.

Todas estas acciones, muestran que los estudiantes y la maestra construyeron el carácter flexible de su curso. Más allá de asumir una forma única y específica de trabajar en línea, al interior de cada grupo de estudiantes se definen las formas y estrategias de interacción, las cuales vinculan experiencias que han vivido en tiempos y espacios anteriores a su curso (Nespor, 1994).

\section{Discusión: la flexibilidad desde los participantes}

Construcción de la flexibilidad en una universidad colombiana pública a distancia con modalidad virtual. René Montero y Judith Kalman. 
En este análisis se exploraron tres de los matices que toma la flexibilidad en un curso en línea. En primer lugar, examinamos la posibilidad que tienen los estudiantes de acceder a la plataforma a cualquier hora y durante cualquier día, mientras lo hagan dentro de los lapsos establecidos por la institución. En segundo lugar, revisamos la posibilidad que tienen los estudiantes de reconfigurar los tiempos de desarrollo de las actividades de acuerdo con el trabajo que vienen desarrollando. Por último, señalamos que aun cuando los estudiantes tengan la posibilidad de acceder a la plataforma a desarrollar sus tareas dentro de lapsos amplios establecidos institucionalmente, sus compañeros pueden excluir sus contribuciones del trabajo grupal.

Los datos derivados de este análisis representan una fuente de información y soporte empírico para cuestionar las formulaciones que consideran la flexibilidad como una característica inherente a la educación a distancia (Daniel, 2016), y que se sostienen en premisas como que los estudiantes tienen la posibilidad de organizar sus tiempos para interactuar con los demás participantes (UNESCO, 2002; OCDE, 2015; Cabero, 2016; Rodríguez, 2016). Más allá de las posibilidades que se encuentran en los discursos oficiales, se mostró que el carácter flexible es una construcción social entre los integrantes del equipo, quienes articulan los lineamientos institucionales con sus expectativas sobre lo que es estudiar en línea, trabajar en equipo, producir una tarea y construir conocimiento. Estos hallazgos muestran la necesidad de continuar estudiando cómo suceden estas construcciones en otros casos.

Como se mostró en los datos, los estudiantes tomaron decisiones sobre los procesos que se llevan a cabo en el aula (Salinas 2004), en este caso sobre las formas de trabajar en línea y sobre la participación que tienen en el desarrollo de las tareas independientemente de la posibilidad de acceso permanente con que cuentan. Esto lo hicieron al margen de las formulaciones, oficiales e institucionales, sobre la flexibilidad de tiempos para realizar los trabajos que tienen en el curso que desarrollan.

Sin embargo, esta aproximación a las interacciones también nos ha permitido explorar elementos adicionales que se conectan con la forma en que se construye el carácter flexible del curso. Por una parte, nos acercamos al tipo de acciones que adelantan los estudiantes en cada uno de sus turnos, y, por otra, pudimos evidenciar la forma que tomó el carácter colaborativo de la elaboración del documento solicitado por la maestra. Mientras ella esperaba socialización de aportes y discusiones para construir consensos, el producto final se decidió mediante la postulación que hizo Julián de su trabajo y la aceptación de dos de sus compañeros.

Si bien los estudiantes tienen la posibilidad de acceder a la plataforma en tiempos y espacios distintos, de acuerdo con sus situaciones laborales y personales (Rodríguez, 2016), esto no supone que cuando participan lo hagan con discusiones académicas. Por el contrario, en los turnos de los estudiantes encontramos acciones como anunciar entregas, emitir juicios sobre los aportes de los demás y felicitaciones ("muy bien por su trabajo", "me gustó su trabajo", "lo felicito por su trabajo" o "su trabajo está muy bien sustentado"). Esta situación muestra que en los foros no necesariamente suceden el tipo de interacciones que esperan los docentes (Gros y Adrian, 2004) y que habría que detener la mirada en el tipo de consignas que construyen los maestros y en el acompañamiento frente a las actividades que deben desarrollar los estudiantes (García y Pineda, 2010).

Construcción de la flexibilidad en una universidad colombiana pública a distancia con modalidad virtual. René Montero y Judith Kalman. 
En este sentido es importante continuar indagando las formas en que suceden los procesos educativos a distancia. Por ejemplo, una de las indicaciones que tenía la maestra en la guía de actividades era que los estudiantes debían realizar aportes pertinentes para el desarrollo del trabajo. Sin embargo, no especificó qué era lo pertinente, ni de qué forma esperaba que los estudiantes construyeran los aportes colectivos que debían realizar en el foro.

Así mismo, es relevante preguntarse acerca de las formas en que los alumnos trabajan los contenidos en el aula en línea, cuando menos en tres aspectos derivados del análisis.

En primer lugar, los estudiantes enviaron en el foro algunos documentos con entregas de sus tareas; sin embargo, no hay registro del proceso de elaboración de éstas, de modo que es posible que los trabajos individuales hayan sido elaborados en espacios diferentes a los que ofrece la plataforma. Esto abre interrogantes por las fuentes de consulta que ellos usaron para elaborar sus tareas o las interacciones que tuvieron con otras personas, ajenas a su curso, que los pudieron orientar.

En segundo lugar, se encontró que la retroalimentación entre pares que sucedió en el aula en línea se remitió a felicitaciones o comentarios del orden de "me gusta su trabajo", sin que se hubiera mencionado algún argumento, desde la disciplina biológica en este caso, que permitiera hacer algún ajuste o ampliación de los documentos entregados. Esto sugiere que para próximos análisis puede ser relevante contactar y entrevistar directamente a los estudiantes, para conocer los criterios que suelen tener en cuenta al momento de comentar el trabajo de sus compañeros, pues de esto tampoco hay un registro en el aula que se analizó.

En tercer lugar, se encontró una baja frecuencia de participaciones de la maestra en el acompañamiento a la elaboración de los productos de los alumnos. En los intercambios presentados, las intervenciones de la docente estuvieron centradas en motivar a sus estudiantes a involucrarse en el desarrollo de las tareas, en orientar algunos aspectos disciplinares de la construcción del producto solicitado y en promover el trabajo en grupo. No obstante, de acuerdo con los hallazgos hay que volver la mirada hacia las asignaciones de tiempos y alumnos que reciben los profesores para atender sus cursos en línea. Los alumnos que ingresan a esta modalidad de estudios requieren trabajar con sus maestros en cuanto al manejo de la plataforma, las estrategias de interacción con sus compañeros y la organización del tiempo para cumplir con las actividades. Aunque los aspectos mencionados no corresponden a la enseñanza de una disciplina es especial, forman parte del trabajo conjunto que se construye durante todo un programa de estudios; más aún cuando es posible que muchos de ellos no hayan tenido experiencias formativas anteriores en línea. En consecuencia, esta investigación muestra la necesidad de considerar el tipo de acompañamiento que se puede hacer y el tiempo que se puede dedicar al trabajo en aula cuando un docente cuenta con asignaciones de estudiantes elevadas, como en el caso analizado.

En cuanto a la forma en que los estudiantes desarrollan el componente colaborativo de la tarea asignada, nuestros datos mostraron que en este grupo la colaboración, que de acuerdo con las indicaciones de la maestra debía reflejarse en discusiones en el foro para construir el producto solicitado, se entendió como compartir alguna opinión frente a las tareas de los demás compañeros y finalmente expresar si se aceptaba o no el trabajo de uno de ellos para ser entregado a nombre de los demás.

Construcción de la flexibilidad en una universidad colombiana pública a distancia con modalidad virtual. René Montero y Judith Kalman.

Página 21 de 26 
Estos resultados muestran que hay una distancia importante entre los modos en que los estudiantes y maestros construyen maneras de colaboración y participación en los espacios digitales y los postulados acerca del trabajo colegiado en línea.

Si bien se promueve a la colaboración como una de las características de la formación a distancia (Gunawardena \& Zittle, 1997; Harasim et al., 2000; Garrison, 2006), nuestros datos permiten relativizar y discutir afirmaciones que señalan que el uso de estas tecnologías transforma los enfoques tradicionales de la educación (Rama, 2016). En los ejemplos presentados, se ilustra cómo los turnos de los estudiantes se enfocaron en hacer entregas de las tareas, y los de la maestra en comentar y orientar a sus alumnos.

La docente registró en la guía de actividades su expectativa frente a la forma en que los alumnos debían elaborar el producto final señalando que:

(1) debían hacer las tareas de forma individual y compartirlas en el foro

(2) debían revisar y comentar en el foro el trabajo enviado por los demás compañeros

(3) debían discutir en el foro sus trabajos y construir un único documento con las tareas solicitadas.

La descripción y el análisis de los datos mostraron que los estudiantes realizaron entregas que no contenían la totalidad de tareas solicitadas y que sus discusiones en el foro se limitaron a felicitaciones y reconocimientos formales de los trabajos de los demás, sin discusiones disciplinares. Inclusive, dos de ellos aceptaron entregar la tarea de Julián como trabajo grupal, sin dialogar sobre sus virtudes o defectos frente a las otras propuestas y, por último, Julián tomó la decisión de no incluir los aportes de Juan en el documento que entregó a la maestra, aun cuando Juan hubiera enviado su aporte dentro del lapso en que la actividad podía desarrollarse.

\section{Conclusiones}

Consideramos que estos resultados dirigen la mirada a la relevancia del análisis del uso del lenguaje en las intervenciones que los estudiantes tienen en los foros en línea, para acercarse a comprender algunas formas en que se vive la educación a distancia directamente desde los participantes. Como mostramos, los estudiantes usan sus mensajes para diversas acciones entre ellas, anunciar entregas, o reconocer el trabajo de los demás, pero no hay evidencia de las formas en que construyen acuerdos sobre los tiempos en que deben hacerse los envíos de los aportes, ni de los criterios que se van a tener en cuenta para decidir cuáles aportes serán incluidos en el trabajo que se entregará a la docente, ni de intercambios disciplinares o discusiones sobre el contenido académico de las tareas a entregar.

Aunque se ha reportado en la literatura que los estudiantes realizan distintos tipos de intervenciones orientadas al manejo de contenidos disciplinares (Anderson \& Kanuka, 1997; Gros y Adrian, 2004), es importante adelantar análisis detallados que muestren, desde una perspectiva del lenguaje (Guerrero y Kalman, 2010; Kalman y Hernández, 2013), las acciones con las que docentes y estudiantes construyen conocimiento en los espacios en línea.

Construcción de la flexibilidad en una universidad colombiana pública a distancia con modalidad virtual. René Montero y Judith Kalman. 
Así mismo, los análisis dejaron ver un aspecto relevante en cuanto al uso de los foros en el curso. En teoría, estos espacios se piensan para sostener discusiones e intercambios de opiniones frente a un tema determinado (Moodle, 2015; Chávez, Del Toro, López, 2017). No obstante, hay diferencias entre las ideas que se tienen desde el diseño de los espacios y el uso que se hace de los mismos (Lefebvre, 2013). En el caso analizado, la maestra solicitó a los alumnos que hicieran un trabajo colaborativo y que para ello emplearan el foro; esto implicaba realizar las tareas individuales, socializarlas, discutirlas, comentarlas con sus compañeros, y construir colectivamente un documento con el trabajo del equipo. Los resultados mostraron que más allá de los diseños y las indicaciones, los usuarios construyen usos de los espacios de acuerdo con sus requerimientos, expectativas e ideas. Aquello que la maestra denominó un trabajo colaborativo, significó para los alumnos postular una tarea individual como trabajo de grupo y esperar que los demás apoyaran la propuesta.

Finalmente queremos resaltar que las consignas sobre la educación a distancia, que normalmente se movilizan a través de los discursos de las agencias internacionales e institucionales, tales como que se trata de espacios colaborativos (Unesco, 2002; OCDE, 2015, Rama, 2016), que promueve el acceso a la educación (Cabero, 2016, Rodríguez 2016) o que ofrece alternativas diferentes a las de los programas presenciales (UNESCO, 2002; Rama 2016), representan descripciones de posibilidades que pueden ser examinadas a la luz de los datos empíricos de las interacciones que se sostienen entre los participantes del aula.

De esta manera, será posible tener una aproximación más detallada a los matices que dichas consignas tienen cuando llegan a las aulas digitales, así como a la necesidad de analizar las experiencias de enseñanza y aprendizaje en estos espacios. Para el caso de este estudio, es importante considerar que la existencia de programas educativos a distancia, no indica por sí misma que las practicas educativas sean flexibles (Salinas, 2004).

Comprendemos que la flexibilidad es una construcción compleja que involucra aspectos como el manejo del tiempo, la posibilidad de conectarse a los cursos desde cualquier lugar con acceso a internet, y la definición de estrategias de estudios de acuerdo con los requerimientos de cada estudiante. Sin embargo, en este artículo se ofreció un acercamiento detallado a la flexibilidad en el manejo del tiempo, lo cual no desconoce los demás aspectos asociados con el carácter flexible de un curso en línea. Por el contrario, la investigación deja ver la necesidad de continuar estudiando esta modalidad educativa con el fin de ampliar nuestro conocimiento al respecto.

\section{Agradecimientos}

Se agradece al Consejo Nacional de Ciencia y Tecnología de México - CONACYT y a la Universidad Nacional Abierta y a Distancia de Colombia - UNAD.

Presentación del Artículo: 9 de julio de 2018

Fecha de aprobación: 24 de septiembre de 2018

Fecha de publicación: 31 de octubre de 2018

Construcción de la flexibilidad en una universidad colombiana pública a distancia con modalidad virtual. René Montero y Judith Kalman. 
Montero, R. y Kalman J. (2018). Construcción de la flexibilidad en una universidad colombiana pública a distancia con modalidad virtual. RED Revista de Educación a Distancia, 58. Consultado el (dd/mm/aaaa) en http://www.um.es/ead/red/58/montero_kalman.pdf

\section{Financiación}

Esta investigación no ha recibido ninguna subvención específica de los organismos de financiación en los sectores públicos, comerciales o sin fines de lucro.

\section{Referencias}

Anderson, T., \& Kanuka, H. (1997). On-Line Forums: new platforms for professional development and Group Collaboration. JCMC [online], 3 (3).

Andresen, M. (2009). Asynchronous discussion forums: Success factors, outcomes, assessments, and limitations. Journal of Educational Technology \& Society, 12(1), 249-257.

Austin, J. (1955). Cómo hacer cosas con palabras. Escuela de Filosofía. Universidad ARCIS.

Barrera, R., Montaño, R., y Marín, P. (2017). Una intervención interactiva por medio de foros en el aprendizaje colaborativo. Edutec. Revista Electrónica de Tecnología Educativa, (62), 73-82.

Bejarano, I., y González, H. (2017). Ser profesionales a distancia. Aproximaciones y respuestas para estudiantes no iniciados en cuanto a la virtualización de la Educación Superior. Revista Científica de la UCSA, 4(2), 63-66.

Blommaert, J. (2005). Discourse. Cambridge: Cambridge University Press.

Blommaert, J., \& Dong, J. (2010). Ethnographic fieldwork: A beginner's guide. Multilingual Matters.

Cabero, J. (2016). La educación a distancia como estrategia de inclusión social y educativa. Revista Mexicana de Bachillerato a Distancia, 15, 8-14.

Castanheira, M. (2001). Interactional Ethnography: an approach to studying the social construction of literate practices. Linguistics and Education, 11(4), 353-400.

Chávez, J., Del Toro, M., y López, O. (2017). Blog, Correo Electrónico y Foros Temáticos: Su Uso, Dominio y Actitud en Estudiantes de Educación Medio Superior de México. Hamut' ay, 4(2), 45-54.

Concha, C. (2014). Educación y Aprendizaje a lo Largo de la Vida: Desafíos para América Latina y el Caribe. En UNESCO. (2014). Apuntes Educación y desarrollo post 2015. Santiago de Chile.

Croxton, R. (2014). The role of interactivity in student satisfaction and persistence in online learning. Journal of Online Learning and Teaching, 10(2), 314-325.

Daniel, J. (2016). Making sense of flexibility as a defining element of online learning. Athabasca University.

Dávila, A., Ruiz, C., y Francisco, J. (2013). Modelo tecno-pedagógico para la implantación de la modalidad semi-presencial en la educación universitaria. Revista Educare, 17(3), 115-140.

García, L. (2009). Concepción y tendencias de la educación a distancia en América Latina. Centro de Altos Estudios Universitarios de la OEI. España.

Construcción de la flexibilidad en una universidad colombiana pública a distancia con modalidad virtual. René Montero y Judith Kalman.

Página 24 de 26 
García, B., y Pineda, J. (2010). La construcción de conocimiento en foros virtuales de discusión entre pares. Revista mexicana de investigación educativa, 15(44), 85111.

Garrison, D. (2006). Online collaboration principles. Journal of Asynchronous Learning Networks, 10 (1), 25-34.

Gee, J. (2005). An introduction to discourse analysis, theory and method. London: Routledge.

Gee, J. (2014). An introduction to discourse analysis: Theory and method. Routledge.

Green, J., Dixon, C., \& Zaharlick, A. (2005). Ethnography as a logic of inquiry. In J. Flood, J. Lapp, \& J. Jensen. Methods of research on teaching the English language arts (págs. 154-194). New Jersey: LEA.

Gros. B., y Adrián, M. (2004). Estudio sobre el uso de los foros virtuales para favorecer las actividades colaborativas en la enseñanza superior. Education in the knowledge society (EKS), 5(1), 1-10.

Guerrero, I., y Kalman, J. (2010). La inserción de la tecnología en el aula: estabilidad y procesos instituyentes en la práctica docente. Revista Brasileira de Educação, 15(44), 213-229.

Gunawardena, C., \& Zittle, F. (1997). Social presence as a predictor of satisfaction within a computer-mediated conferencing environment. American Journal of Distance Education, 11, 8-26.

Habib, L., \& Wittek, L. (2007). The portfolio as artifact and actor. Mind, Culture, and Activity, 14(4), 266-282.

Harasim, L., Hiltz, S. R., Turoff, M., y Teles, L. (2000). Redes de aprendizaje. Guía para la enseñanza y el aprendizaje en red. Barcelona: Gedisa.

Hardman, F. (2008). The guided co-construction of knowledge. En M. Martin Jones, A. De Mejía, y N. Hornberger, Encyclopaedia of Language and Education (págs. 253-264). Springer.

Heath, S. (1982). Ethnography in education: Defining the essentials. In P. Gilmore, y A. Glatthorn, Children In and Out of School (págs. 33-55). Washington: Center for Applied Linguistics.

Hernández, G. (2005). La comprensión y la composición del discurso escrito desde el paradigma histórico-cultural. Perfiles educativos, 27(107), 85-117.

Hine, C. (2004). Virtual Etnography. London: Sage.

Jaggars, S. (2014). Choosing between online and face-to-face courses: Community college student voices. American Journal of Distance Education, 28(1), 27-38.

Kalman, J. (1996). Joint composition: The collaborative letter writing of a scribe and his client in mexico. Written Communication, 13(2), 190-220.

Kalman, J., y Street, B. (Eds.). (2009). La lectura, escritura y matemáticas como prácticas sociales. Diálogos con América Latina. México, DF.: Siglo XXI Editores y CREFAL.

Kalman, J., y Hernández, O. (2013). Jugar a la escuela con pantalla y teclado. Archivos Analíticos de Políticas Educativas, 21, 1-24.

Kalman, J., y Rendón, V. (2016). Uso de la hoja de cálculo para analizar datos cualitativos. Magis. Revista Internacional de Investigación en Educación, 9(18), 29-48.

Lankshear, C., \& Knobel, M. (2011). New literacies. McGraw-Hill Education (UK).

Lave, J., \& Wenger, E. (1991). Situated Learning. Legitimate Peripheral Participation. Cambridge: Cambridge University Press.

Lefebvre, H. (2013). La producción del espacio. Madrid: Capitán Swing.

Construcción de la flexibilidad en una universidad colombiana pública a distancia con modalidad virtual. René Montero y Judith Kalman. 
Maya, A. (1993). La educación a distancia y la función tutorial. UNESCO. San José.

MOODLE. (2015). Pedagogía. Disponible en línea en https://docs.moodle.org/all/es/Pedagog\%C3\%ADa

Nandi, D., Hamilton, M., \& Harland, J. (2012). Evaluating the quality of interaction in asynchronous discussion forums in fully online courses. Distance Education, 33(1), 5-30.

Nespor, J. (1994). Knowledge in motion: Space, time and curriculum in undergraduate physics and management. Routledge.

Novo, M. (2004). La Educación Ambiental a distancia: su alcance y posibilidades. Biocenosis, 18(1), 79-84.

OCDE. (2015). Competencias más allá de la escuela, síntesis, resumen y recomendaciones políticas. OCDE.

Rama, C. (2016). Modelos de educación a distancia y dinámicas educativas en América Latina. 25-36; En Domínguez, J y Rama, C. (2016). La Educación a Distancia en el Perú - Chimbote: ULADECH Católica, 2013.

Rodríguez, J. (2016). Aproximaciones a la educación a distancia en el Perú. 37-53; En Domínguez, J y Rama, C. (2016). La Educación a Distancia en el Perú Chimbote: ULADECH Católica, 2013.

Ruiz, M. (2016). Análisis pedagógico de la docencia en educación a distancia. Perfiles educativos, 38(154), 76-96.

Salinas, J. (2004). Cambios metodológicos con las TIC. Estrategias didácticas y entornos virtuales de enseñanza-aprendizaje. Bordón, 56(3-4), 469-481.

Silva, J. (2017). Un modelo pedagógico virtual centrado en las E-actividades. RED. Revista de Educación a Distancia, 53. Consultado el (26/01/2018) en http://www.um.es/ead/red/53/silva.pdf

Street, B. (2005). Literacies Across Educational Contexts. Mediating Learning and Teaching. Philadelphia: Caslon, Inc.

UNAD. (2011). Proyecto Académico Pedagógico Solidario. UNAD. Bogotá.

UNESCO. (2002). Aprendizaje abierto y a distancia Consideraciones sobre tendencias, políticas y estrategias. Trilce. Montevideo.

UNESCO. (2017). Aprendizaje abierto y a distancia. Recuperado de http://www.unesco.org/new/es/unesco/themes/icts/lifelong-learning/open-anddistance-learning/

Wertsh, J. (1993). Voces de la mente. Un enfoque sociocultural para el estudio de la Acción Mediada. Madrid: Visor.

Construcción de la flexibilidad en una universidad colombiana pública a distancia con modalidad virtual. René Montero y Judith Kalman. 